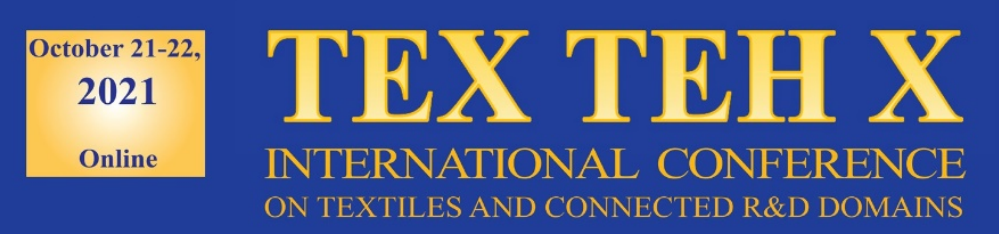

\title{
RESEARCH REGARDING NEW SUSTAINABLE METHODS OF GARMENT PATTERN DRAFTING
}

\author{
DOI: 10.35530/TT.2021.11
}

\begin{abstract}
I.E. Marin
Doctoral School at the “Gheorghe Asachi” Technical University of Iaşi, Romania (E-mail: irina.marin13@yahoo.com)

Abstract: Based on the present need for change in the garment industry, multiple solutions for waste reducing have been established. It has become common to associate fashion with pollution, and this association has its roots in the statistical data that constantly raise red flags regarding the negative impact that our everyday textiles have over the planet. The following paper has been developed under the strict rules that the zero waste pattern drafting has, keeping in mind the design and product finishing matters. Further possible issues have been taken into account whilst defining and shaping the pattern pieces, aiming to obtain a product that is framed by proper aesthetics, fit, zero waste drafting technique and quality finishing. The way in which the waste management can be kept under control from the pattern drafting step is the key to gaining a sustainable clothing item. Apart from the fabric scraps, the time and energy needed for sampling can also be optimized, by using the virtual prototyping opportunity. These two crucial elements, combined, led to garments that can last in time.

The nexus of the garment manufacturing techniques that have increased in popularity lately has been elaborated in the following paper, following the process accordingly, shaping into a number of digital clothing items. The method used can be successfully implemented by clothing manufacturers that wish to adopt a healthy and non pollutive process.
\end{abstract}

Keywords: Consumer's behaviour; efficient cutting; sewing patterns; sustainability; waste management

\section{INTRODUCTION}

Driven by the increasing numbers that point out the negative impact that fashion has over the environment, searching for sustainable approaches within production has become the main concern of the experts working in the field. So far, the consumers were delighted by fashion's colourful and mesmerizing aesthetics, without acknowledging the harm that's being inflicted on the environment for catwalks and mass production to be possible.

It has become widely known that the fashion industry is one of the most pollutive industries in the world, based on the fabric scraps that end up in the landfill, carbon footprint that's generated by production and water poisoning caused by poor waste management. The effects have been publicized enough to catch the public eye and turn consumer's awareness to the disaster that's happening behind the deceitful splendour of fashion.

However, apart from acknowledgement, consumers must be informed, encouraged, directed and advised in regards of taking proper care of their garments, and thus ensure their clothing items can get a growth in longevity. And by doing so, the fast fashion trend will be drastically reduced, from the customer's behalf. By informing the consumers about the damage that they unknowingly and unwillingly make may bring their awareness over the habitual clothing disposal. By encouraging them, the process will have a much greater rate of success. By directing the buyer, one will know and be able to dispose and care for its clothing items accordingly. Last but not least, by advising the consumer in regard to the correct 
methods of caring for its garments leads to a win-win situation: longer lasting clothes contribute to diminishing the chaotic disposal, and thus landfill pollution.

Some producers have also adopted more sustainable methods and kept their mind open when implementing the new approaches within the factories. New sustainable fabrics have been developed, along with sewing threads and natural dyes, which contribute to decreasing the negative impacts of the garment industry over the planet.

Within the designing sphere, the Zero Waste method started to flourish for the past decade. Approaching an ancient manufacturing technique and using creative fabric manipulation methods, it has led to a series of solutions that reduce the fabric scraps, in most cases, completely [1].

This new way of approaching the pattern drafting has been introduced within the $21^{\text {st }}$ century [2], but it dates back in the ancient times, if we were to consider the traditional Kimono - for manufacturing this type of clothing, a rectangular fabric was needed, using it completely and thus, avoiding fabric waste.

Moreover, virtual prototyping software have been developed and launched for use in the past years. These programs aim to assist in sustainable methods, by displaying the final product on the screen. This enables to have a better view of the fitting, pattern accuracy or fabric drape, without having the physical sample manufactured. Important resources such as time and energy are reduced when digitally assembling a clothing item.

This study has been made by analysing the information gathered, a series of testing and simulating the final virtual prototypes developed along the process.

\section{MATERIALS AND METHODS}

\subsection{Materials}

For the present paper, digital resources have been mostly used to display the consumer's perception of what is known as sustainable fashion, its behaviour regarding garment disposal, as well as testing the pattern drafting method and simulating the end result within the $3 \mathrm{D}$ space.

Consumers have been addressed by an online survey, which contained a series of key questions, meant to bring to light the consumer's way of thinking and awareness regarding everyday life textiles.

The patterns have been drafted using the dedicated software Gemini Pattern Editor and garments were simulated by using the CLO 3D virtual fashion software.

The paper has been developed as a result of researching the already existing techniques, analysing the results and spotting the downsides of each experimentation. Theoretical information has been collected from studying current literature, complemented by notes gathered from personal practice.

\subsection{Methods}

\subsubsection{Analysing the consumer's behaviour}

When asked, a number of participants have claimed that they have heard of sustainability within the fashion field. More than a half of them are somewhat familiar to the term, which gives a clue regarding the consumer's information. However, despite being informed, most of them do not hold the proper knowledge of what does sustainability stand for.

The following question gave much clearer information regarding the comprehension of this term: most of them associated sustainable clothing items to high prices. This stands for the fact that quality and price are reliable. Most consumers aim to purchase good quality items 
on lower prices, and that's because they are not informed regarding the costs of manufacturing and producing the fabric, the thread and the garment itself, which also includes labour and shipping. This conclusion stands from the following simple analysis: $60 \%$ of the consumers have heard of "sustainability" before, but only $47 \%$ of them knew what it stands for. Thus, a quantum of $13 \%$ of them is not properly informed nor do they hold the correct pieces of information regarding this matter.

The participants have been asked about the way they dispose of their unwanted garments. This question is meant to offer a clear image of their behaviour. Over $50 \%$ of them hand their clothing to others in need, by donating them to charity or younger family members, friends, acquaintances (figure 1). This gives a clue regarding the way the consumer cares for its garments, assuming that their donated items are still in good condition. This way of disposing seems more comfortable and efficient, but only when it comes to a certain category of garment. Ideally, the clothes that are worn out, torn or have any sort of damage should be disposed at local collecting bins that specialize in sorting and recycling. However, unfortunately that's not the case, since $5 \%$ of the consumers questioned have claimed that they simply throw away their unwanted clothing. This highlights the poor level of information, education of the consumers, as well as the poor fabric waste management.

QUESTION 07 | MULTIPLE CHOICE

How do you dispose of unwanted clothes (choose all that apply)

Answered: $\mathbf{2 0}$ Skipped: 0

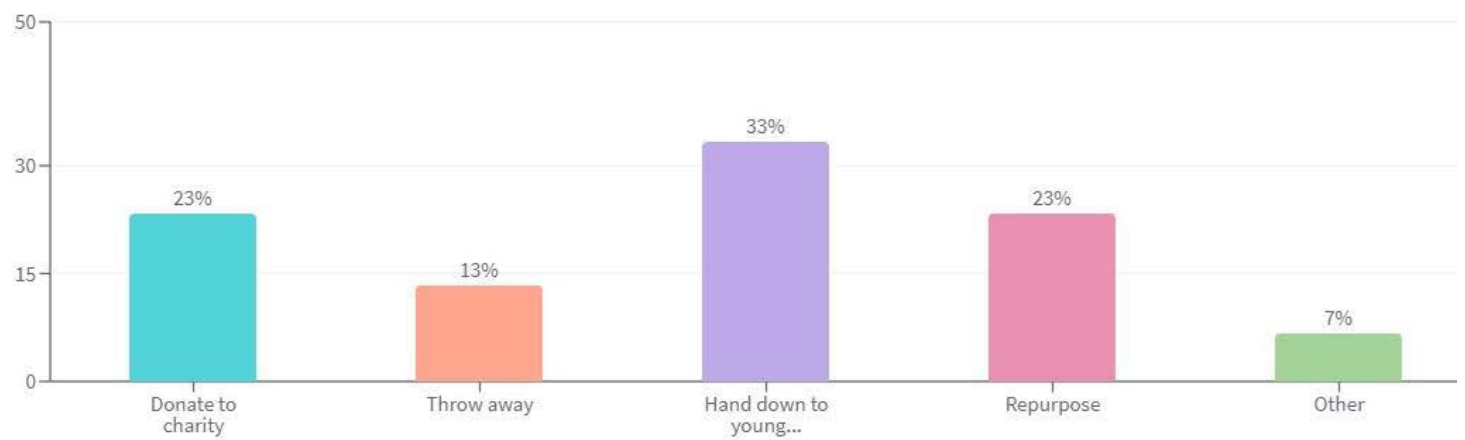

Figure 1. Data collected from the consumer regarding their clothing disposal habits

\subsubsection{Drafting the Zero Waste patterns}

This Zero Waste pattern drafting method consists of altering the pattern shapes of an already existing pattern set. Thus, there's been a transition from a regular fit to a looser fit. This method approaches in a different way the Zero Waste pattern drafting: rather than starting from a simple rectangle that would represent the lay plan, the patterns have been amended in order to fit within and form a lay plan [3]. The pieces have been modified and reshaped, starting from a classic form and obtaining a geometrical configuration. This procedure allowed the direct and evident observations of the differences between classic and zero waste patterns, based on fabric consumption, lay plan efficiency and body fit. Moreover, the final design of each style has been established during the process, in accordance to the pieces' permeability. The process consists in a series of consecutive systematic steps, that can't be skipped nor replaced. 
Simplifying the pattern's geometry consisted in reducing the French curves as much as possible, keeping in mind the intention of creating a clothing item that's more than a square and less than a typical garment piece. In some cases, the already existing curves have been kept in order to serve to a better pattern interpolation and were used as joining edges for other pattern pieces that were further used for practical or aesthetic purposes.

The most crucial step was to define the seam allowances value - pattern nesting is highly influenced by the cutting perimeter. A method studied has revealed the solution of using variable values for seam allowances [4]. However, given the poor aesthetic on the inside of the garment resulted by using such technique, this doesn't stand for unlined garments. Moreover, the sewing technology is being limited to a simple stitch, with possible serged edges. Considering these possible results, all seam allowances that were to be assembled have been defined with constant values.

It's important to mention the fact that in order to avoid having an exaggerated fit, some garments designed have been modelled on the important areas of the body (for example, on the waistline) by using rows of elastic thread. This solution also allowed for the specific pattern pieces to be as wide as it needed to fit and match for an efficient lay plan, acknowledging that they will shape the body by later on. Other shape manipulation techniques have been used, such as pleats on the shoulder line, elasticated cuffs and shirring rows sewn on the waistline, as shown in the figure 2 .

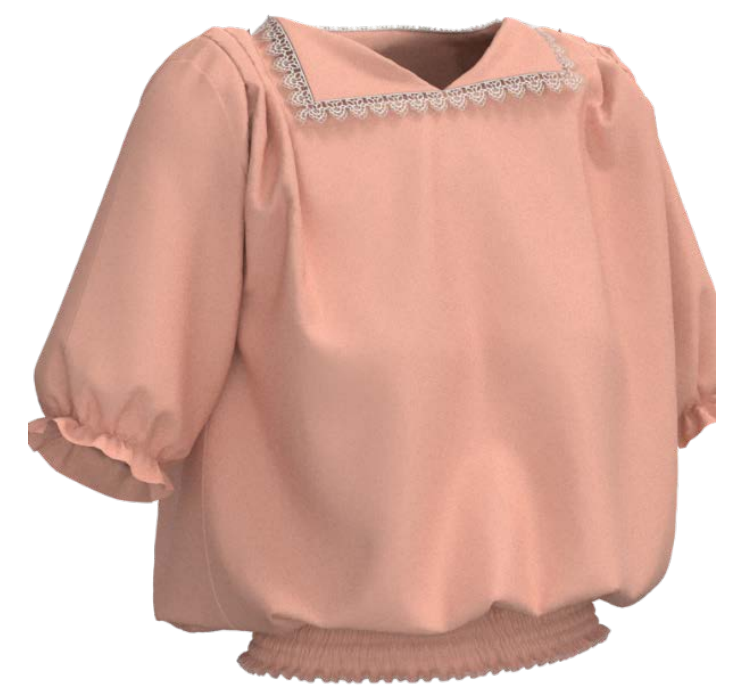

Figure 2. Example of an accurate fitting obtained by using shape manipulation solutions: pleats on the shoulder line, to maintain a regular shoulder length and shape, elastic thread close the sleeve hem, for aesthetic and practical purposes and elastic threads on the waistline

\section{RESULTS AND DISCUSSIONS}

This Zero Waste pattern drafting method has been applied to several clothing items, adapted to the garment's functionality and design. Given the example of a woollen coat, the classic basic patterns have been drafted from scratch. The geometrical patterns have been further developed, in order to gain the required jigsaw placement, and thus result in two different, yet similar pattern sets which have been used for further analysis. The final shapes are shown in figures 3 and 4. 


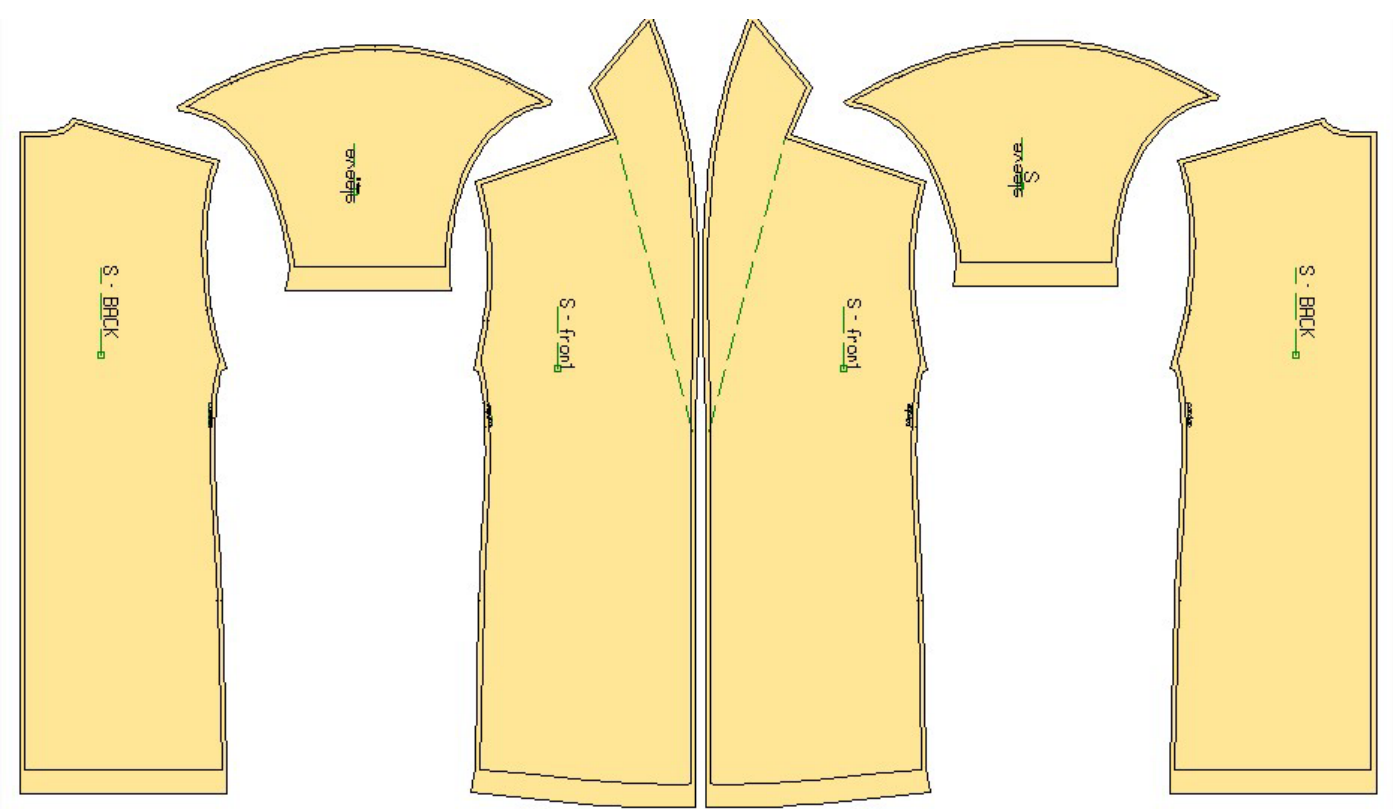

Figure 3. Basic patterns for the classic woollen coat

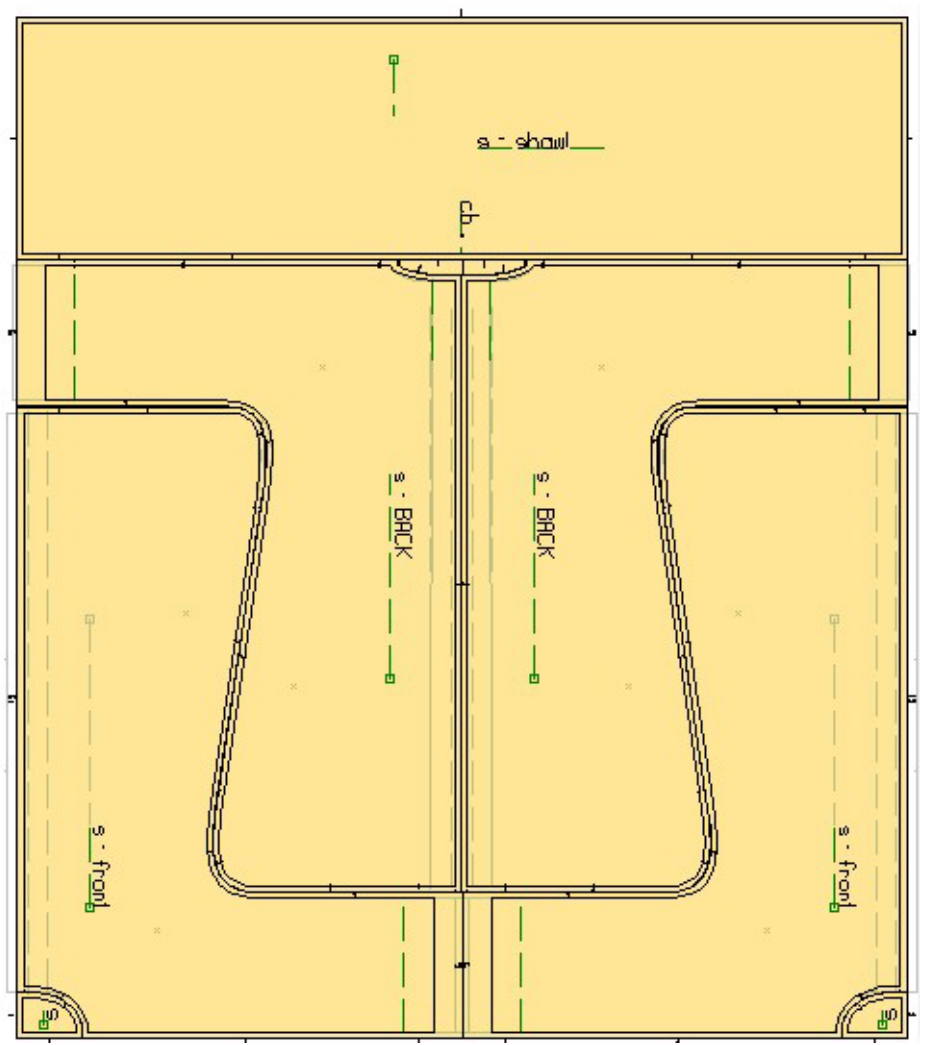

Figure 4. Zero waste patterns for the woollen coat

Once the patterns were defined for both cuts, the next step was to generate individual lay plans, and thus gather the first pieces of information that highlighted the differences in fabric consumption and nesting efficiency. The values obtained have been summed in the table 1. 
Table 1. Differences between two types of patterns for the same clothing item

\begin{tabular}{|c|c|c|c|c|}
\hline \multirow{2}{*}{ Woolen Coat } & $\begin{array}{c}\text { Classic cut } \\
\text { efficiency [\%] }\end{array}$ & $\begin{array}{c}\text { Zero waste cut } \\
\text { efficiency [\%] }\end{array}$ & $\begin{array}{c}\text { Classic cut fabric } \\
\text { rating [m] }\end{array}$ & $\begin{array}{c}\text { Zero waste cut } \\
\text { fabric rating [m] }\end{array}$ \\
\cline { 2 - 5 } & 68.05 & 95.2 & 2.7 & 1.75 \\
\hline
\end{tabular}

Analysing these results brings a clear insight regarding how much the fabric waste can be reduced when approaching different drafting methods.

By examining the efficiency values, it is clear to see that the overall fabric consumption when using zero waste patterns increased by $71,4 \%$, which signifies that the pattern pieces have been joined and placed together with little space between them.

In the same time, the fabric consumption has decreased by $64.8 \%$, meaning that it takes less fabric to cut the zero waste patterns, hence their jigsaw placement. It's important to mention that both pattern sets have been nested on the same fabric width, figures 5 and 6 .

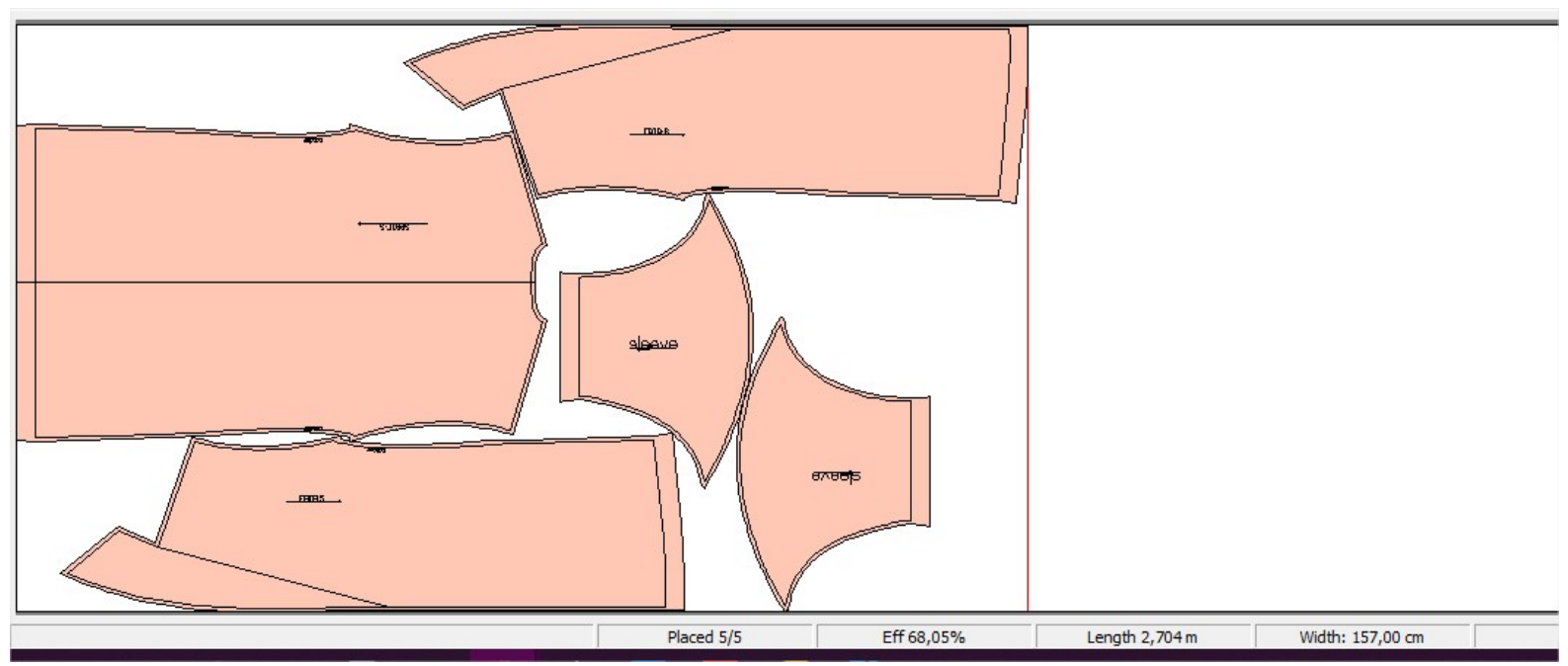

Figure 5. Lay plan for the classic coat patterns

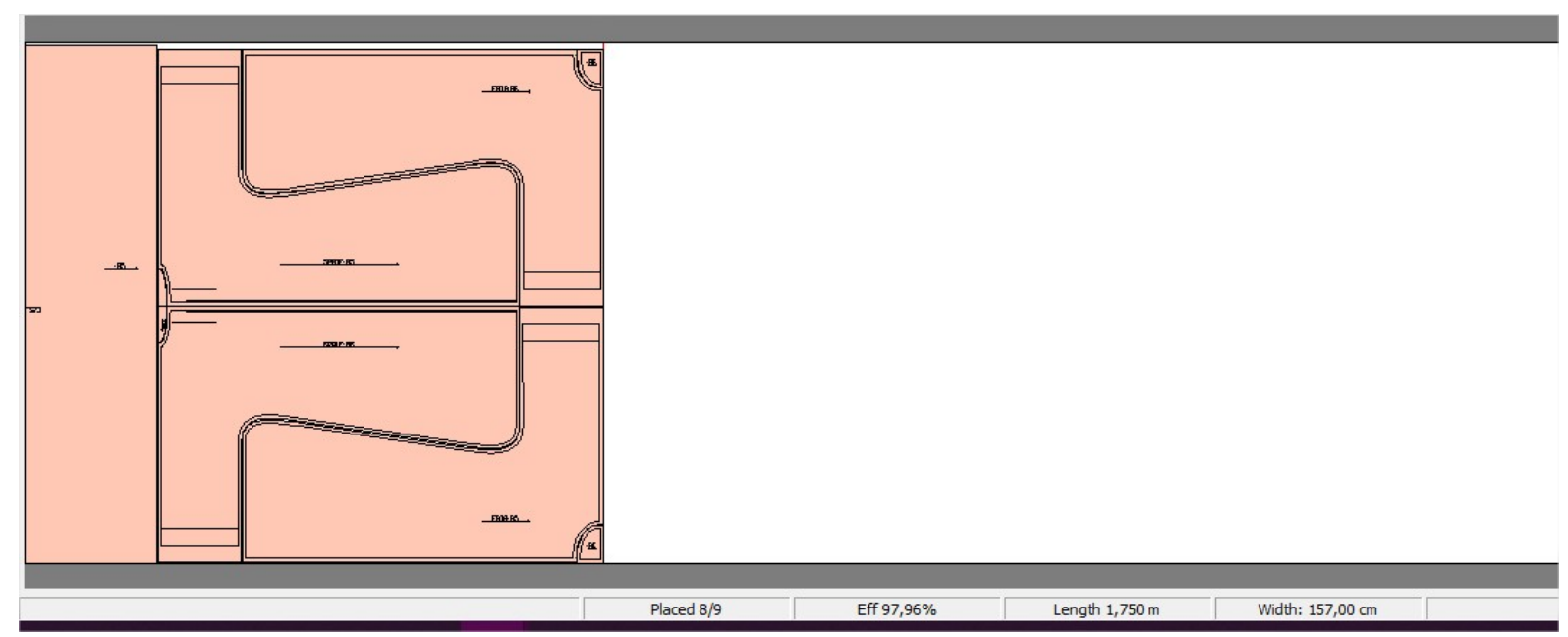

Figure 6. Lay plan for the zero waste coat patterns 
Once the data have been collected, the second part of the process was approached: aesthetics. The garment has been digitally assembled, fitted and finished by using the virtual prototyping software. The avatar's measurements correspond to the Alvanon size 36 EU. The patterns have been drafted for size S (36 EU).

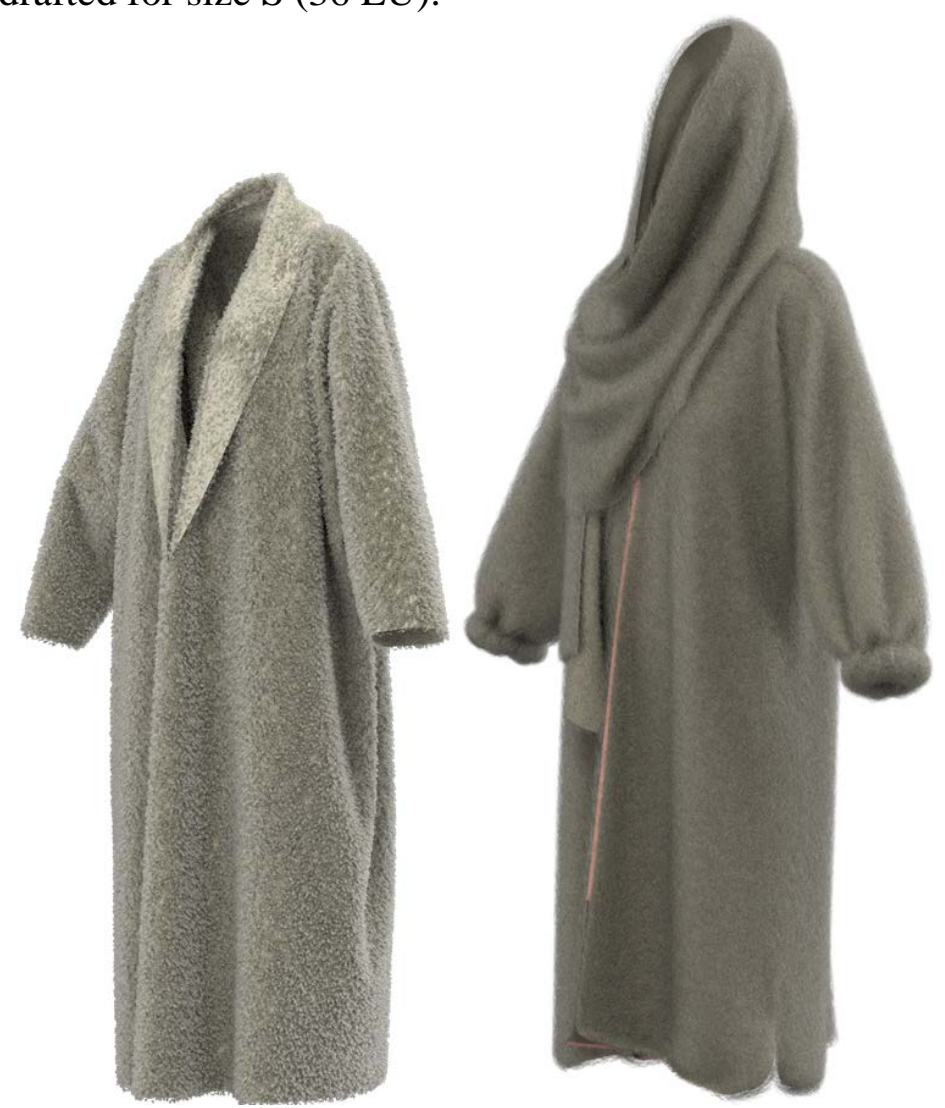

Figure 7. Simulated virtual sample for the woollen coat: classic fit to the left and zero waste fit to the right

\section{CONCLUSIONS}

It takes both creativity and know-how to develop zero waste pattern sets for almost any type of garment item. Apart from establishing the final design during the pattern drafting process, the finishing and manufacturing technology must also be kept in mind. Each step is framed into a series of future possibilities; therefore, the technological and design matters are co-dependent and complementing each other.

The results haven't been regarded as virtual prototypes, but as real physical samples that can be worn, and thus must have clean finishing and true practical purposes, above all. Edges finishing, assembling and hem edges - all of these matters have been taken into account during the drafting process and whilst defining the seam allowances.

Applying this method has proven to be an efficient solution for reducing fabric waste and, also, designing unique clothing that hold the possibility to refresh the fashion market across the world.

\section{REFERENCES}

[1] Gwilt, A., Zero Waste Fashion Design: Approaches and strategies to reduce textile waste, Sheffield Halam University, UK, 2016 
[2] Jalil, M., Hosseini, S., Quest for the fashion market: Zero Waste skirt design as a solution toward sustainable pattern making, In: The Chitrolekha Journal on Art and Design, 2020, 4, 2, 2, https://dx.doi.org/10.21659/cjad.41.v4n205

[3] Rissanen, T., McQuillian, H., Zero waste fashion design (Vol. 57), In: Bloomsbury Publishing, 2016

[4] Ejeimi, S., Grading zero waste design using digital and virtual methods, Kansas State University, USA, 2020 